\title{
Single and multiple optical switches that use freestanding silicon nanowire waveguide couplers
}

\begin{abstract}
Yuta Akihama and Kazuhiro Hane
Silicon photonic devices consisting of nanowire waveguides are a promising technology for on-chip integration in future optical telecommunication and interconnection systems based on silicon-large scale integration fabrication. However, the accommodation of variable optical components on a chip remains challenging due to the small size of microchips. In this study, we investigated the characteristics of a microelectromechanical silicon nanowire waveguide switch with a gap-variable coupler. Due to its capacitive operation, the proposed waveguide switch consumed negligible power relative to switches that use a thermo-optical effect and carrier injection. The proposed switch was characterized using analyses based on coupled-mode theory for rectangular waveguides, as well as a simulation using the finite difference time domain method. A $2 \times 2$ single switch with an improved configuration and a $2 \times 6$ multiple switch composed of the $2 \times 2$ switches was designed and fabricated by a combination of electron beam lithography, fast-atom beam etching and hydrofluoric acid vapor sacrificial etching. The properties of the switches were measured and evaluated at a wavelength of $1.55 \mu \mathrm{m}$.
\end{abstract}

Light: Science \& Applications (2012) 1, e16; doi:10.1038/Isa.2012.16; published online 22 June 2012

Keywords: microelectromechanical systems; optical switch; silicon photonics

\section{INTRODUCTION}

Submicron-scale silicon waveguide circuits are a promising technology for optical telecommunication and interconnection applications. ${ }^{1}$ The monolithic fabrication of silicon waveguides and silicon electronics is useful for future integration in opto-electronic systems. Due to the high refractive index of silicon $(\sim 3.5$ at a wavelength of $1.5 \mu \mathrm{m})$, silicon waveguide circuits can be miniaturized to be several orders of magnitude smaller than silica waveguide circuits. Several types of circuits that employ submicron-scale silicon waveguides, such as waveguide splitters/couplers and micro-rings, ${ }^{2-4}$ have been reported. In addition, a waveguide switch that uses a thermo-optical effect ${ }^{5}$ and an ultra-fast silicon waveguide light modulator based on changes in the refractive index of silicon by carrier injection ${ }^{6}$ have been reported.

Recently, submicron-scale waveguide switches for optical path changes using the nano-mechanical motions of electrostatic actuators have been reported. ${ }^{7}$ Due to the very low power consumption associated with capacitive operation, this technology suits the large-scale integration and reduced energy consumption requirements of telecommunication systems. ${ }^{8-16}$ Among these waveguide circuits, coupler switches have attracted interest due to the use of non-contact low-loss mechanisms. ${ }^{13,15}$ A coupler switch using InP waveguides was operated by applying electrostatic forces between the freestanding waveguides. ${ }^{13}$ In the case of a parallel-electrode actuator, a coupler gap smaller than two-thirds of the initial gap is not consistently controllable due to force instability. Using an in-plane comb-drive actuator, stable low-voltage operation has been realized for silicon waveguides. ${ }^{15}$ However, the characteristics of a freestanding submicron-wide silicon waveguide coupler switch have not been theoretically or experimentally analyzed in detail. Moreover, the feasibility of a matrix switch that employs coupler switches has not been investigated.

In this paper, the design and fabrication of an improved submicronscale silicon waveguide coupler switch that uses an ultra-small electrostatic comb-drive actuator are reported, together with theoretical analyses. The fabrication and characteristics of multiple switches that employ the coupler switch elements are also reported. In the fabricated single switch, periodic change in the coupling as a function of the gap was clearly observed. The switching conditions were experimentally demonstrated using the gap-variable mechanism. The large-scale switch was quantitatively investigated to determine the feasibility of a matrix switch consisting of the proposed coupler switches. The switch characteristics, such as the port isolation and cross talk, were experimentally evaluated.

\section{PRINCIPLE AND DESIGN}

Figure 1a presents the basic structure of the proposed waveguide coupler switch. The coupler consisted of two freestanding silicon waveguides, which were suspended in air. Two waveguides were located within their region of interaction (100-500 nm). The gap between the waveguides was varied in the horizontal plane, while the waveguides remain parallel. The shape of the coupler was symmetric with respect to the center line between the waveguides to avoid asymmetric electromagnetic distribution of light waves, which was different from the previous design. ${ }^{15}$ One of the waveguides of the coupler was connected to an electrostatic comb-drive actuator with 


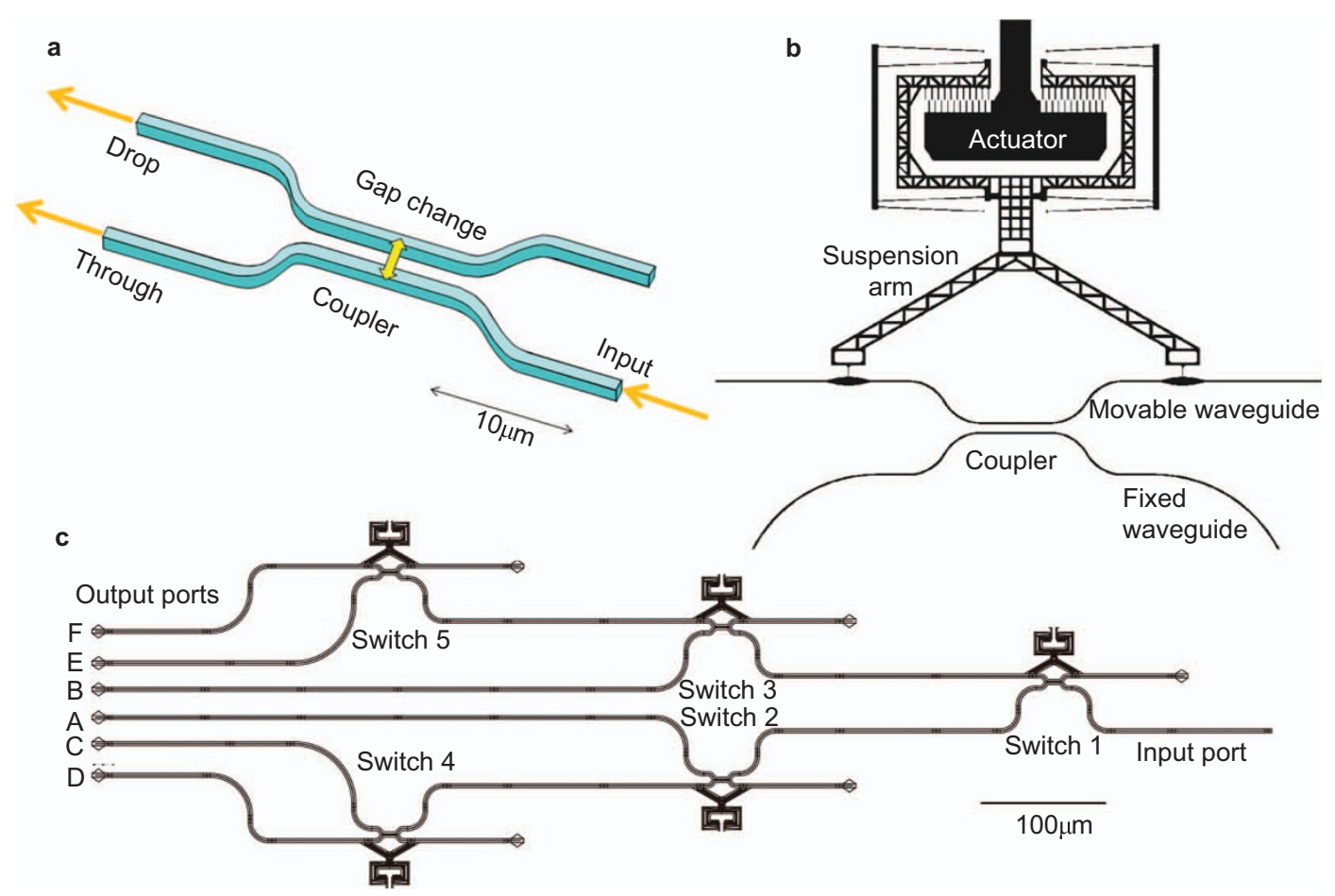

Figure 1 (a) General structure of the silicon freestanding nanowire waveguide coupler switch with a gap-variable mechanism; (b) schematic diagram of the coupler switch with an electrostatic comb-drive actuator; (c) schematic diagram of a $2 \times 6$ multiple switch consisting of $2 \times 2$ waveguide coupler switches.

two suspension arms, as schematically illustrated in Figure 1b. The movable waveguide was translated by the actuator. Although two actuators were used in the previous design, ${ }^{15}$ the actuator structure was improved to assure the parallel motion and avoid a complex structure, as shown in Figure 1b. The suspension arm and the waveguide were connected by an elliptical low-loss waveguide bridge. ${ }^{17}$ The loss of the elliptical waveguide bridge was shown to be less than $0.1 \mathrm{~dB}$. The other waveguide of the coupler was also freestanding in air and was fixed to the substrate via the elliptical waveguide bridges. By applying a voltage to the comb-drive actuator, the movable waveguide was translated to decrease the gap, and thus, the strength of the coupling between the waveguides increases.

We also designed and fabricated a large scale $2 \times 6$ switch using the waveguide coupler switch elements, as shown in Figure 1c. The waveguide coupler switches were connected in series and in parallel. The individual waveguide coupler switch elements functioned as $2 \times 2$ switches. By applying voltages to the respective coupler switches, different optical paths were connected between the inputs and outputs. The switching architecture was similar to a switch consisting of micromirror $2 \times 2$ switches. ${ }^{18,19}$ The area of the individual coupler switch

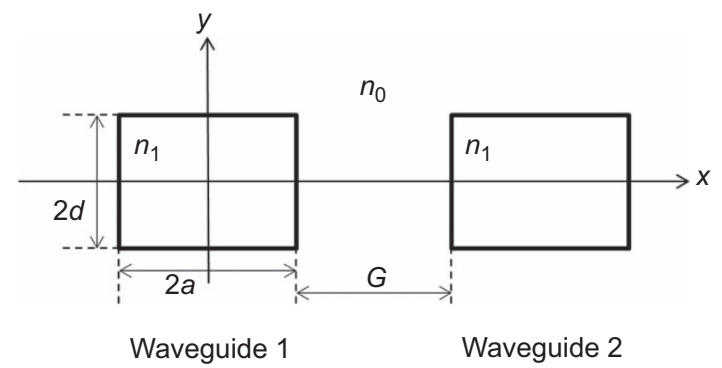

Figure 2 Cross-sectional schematic diagram of the waveguide coupler. was as small as $100 \mu \mathrm{m} \times 100 \mu \mathrm{m}$, and thus, a matrix switch consisting of 100 switches can be installed in a 1-mm $\times 1-\mathrm{mm}$ area, for example.

\section{THEORETICAL APPROACH}

Waveguide couplers have been studied for many years since the early development of waveguide optics. A rectangular waveguide coupler is a basic coupler for studying the fundamental characteristics of a general coupler and for obtaining analytical equations that describe waveguide coupling. ${ }^{20,21}$ In the proposed coupler switch, the cross-sections of the silicon waveguides were assumed to be rectangular, as schematically illustrated in Figure 2. The two waveguides had identical dimensions with a width of $2 a$ and a thickness of $2 d$. The distance between the centers of the two waveguides was $D$, and the air gap between the waveguide walls was $G$, as shown in Figure 2 . The refractive indices of the waveguide and the surrounding air were $n_{1}$ and $n_{0}$, respectively. In the proposed coupler switch, the gap $G$ between the two waveguides was varied along the $x$ axis by the electrostatic combdrive actuator. Therefore, the coupling coefficient was obtained in a manner similar to that for a basic rectangular waveguide coupler with the gap as a parameter. The coupling coefficient was obtained using the following equation: ${ }^{21}$

$$
\chi=\frac{k^{2}\left(n_{1}^{2}-n_{0}^{2}\right)}{\beta} \frac{\left(k_{\mathrm{x}} a\right)^{2}\left(\gamma_{\mathrm{x}} a\right)^{2}}{\left(1+\gamma_{\mathrm{x}} a\right) v^{4}} \exp \left(-\gamma_{\mathrm{x}} G\right)
$$

where $k_{\mathrm{x}}$ and $\gamma_{\mathrm{x}}$ are wave number and asymmetric constant, respectively, of the refractive index distributions along the $x$ axis. The symbols $k$ and $\beta$ represent the wave number in a vacuum and a propagation constant along the $z$ axis, respectively. The normalized frequency is given by $v=k n_{1} a \sqrt{2 \Delta}$ with the refractive index difference given by $\Delta=\left(n_{1}^{2}-n_{0}^{2}\right) / 2 n_{1}^{2}$. Although silicon has a high refractive index, this approach was used to obtain a simple approximation of the coupling coefficient. 

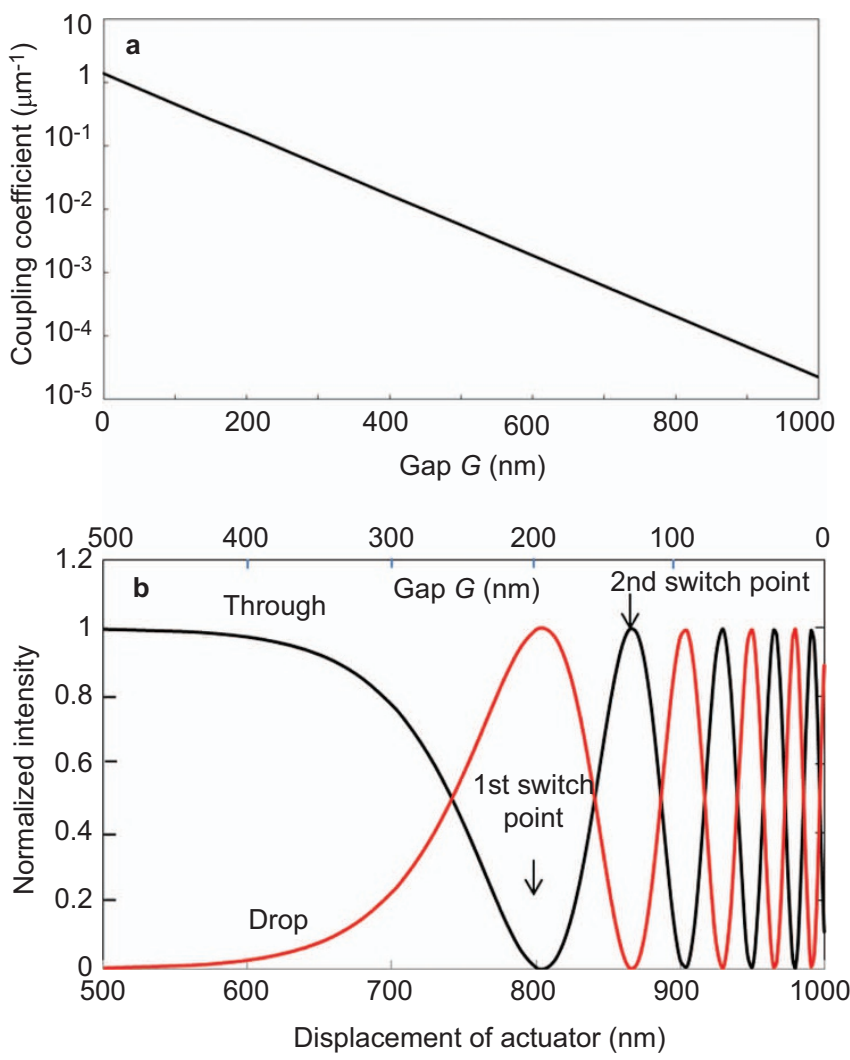

Figure 3 (a) Coupling coefficient calculated as a function of gap G; (b) normalized light intensities at the through and drop ports of the coupler switch calculated as a function of the actuator displacement and gap $G$ between the waveguides.

The output intensity $I_{1}$ of the transmitted light in waveguide 1 at the through port and the output intensity $I_{2}$ of the transmitted light in waveguide 2 at the drop port were expressed, respectively, using the following equation:

$$
I_{1}=I_{0} \cos ^{2}\left(\chi L_{\mathrm{z}}\right) \text { and } I_{2}=I_{0} \sin ^{2}\left(\chi L_{\mathrm{z}}\right)
$$

where $I_{0}$ is the input light intensity and $L_{\mathrm{z}}$ is the coupler length. The intensities changed sinusoidally with an increase in the coupler length or coupling coefficient.

We selected a waveguide width to allow for only the single lowest mode of the waveguide, and we assumed that the mode profile was that of the lowest transverse electric (TE)-like mode. The width of the waveguide was designed to be $400 \mathrm{~nm}$. The refractive index of silicon was 3.47 at a wavelength of $1.55 \mu \mathrm{m}$. The height of the waveguide was $260 \mathrm{~nm}$, which was equal to the thickness of the top layer of a silicon on insulator (SOI) wafer. Under our experimental conditions, the values of the parameters were as follows: $\Delta=0.46, a=200 \mathrm{~nm}$, $k_{\mathrm{x}}=7.6 \times 10^{6} \mathrm{~m}^{-1}, \gamma_{x}=1.1 \times 10^{7} \mathrm{~m}^{-1}, \beta=8.7 \times 10^{6} \mathrm{~m}^{-1}$ and $v=2.7$.

The calculated coupling coefficient as a function of gap $G$ between the waveguides is shown in Figure 3a. The coupling coefficient decreased exponentially. Therefore, the coupling was changed by varying the coupler gap. The value of the coupling was approximately $10^{-5} \mu^{-1}$ at $G=1000 \mathrm{~nm}$, which was sufficiently small to be considered a switched-off condition. We designed the gap of the coupler switch to be varied within a range of less than $1000 \mathrm{~nm}$.

Figure $3 \mathrm{~b}$ shows the normalized light intensities at the through and drop ports of the coupler switch calculated as a function of $G$ by assuming that the coupling length of the waveguide was $10 \mu \mathrm{m}$ in equation (2). Because the gap was varied by the displacement of the actuator from an initial gap of $1000 \mathrm{~nm}$, the displacement of the actuator is also shown in Figure $3 \mathrm{~b}$. As the gap decreases, the intensity at the through port periodically changes, whereas the intensity at the drop port periodically varied to be out of phase with the intensity at the through port. Two main switch points were found in the displacement region: one was at approximately $805 \mathrm{~nm}(G \approx 195 \mathrm{~nm}$, the first switch point), and the other was at $865 \mathrm{~nm}(G \approx 135 \mathrm{~nm}$, the second switch point). Therefore, two switching conditions were applied: one condition was used to change the waveguide position from the zerodisplacement point $(G=1000 \mathrm{~nm})$ to the first switch point with a displacement of approximately $805 \mathrm{~nm}$, and the other condition was used to shift from the first switch point to the second switch point with a displacement of approximately $60 \mathrm{~nm}$. A comparison of the two switch points indicates that the tolerance of the actuator displacement at the second switch point was smaller than that at the first point.

The normalized light intensities at the through and drop ports were also calculated at the first switch point as a function of wavelength. The maximum extinction ratio was designed at a wavelength of $1.55 \mu \mathrm{m}$. An extinction ratio of $30 \mathrm{~dB}$ was obtained in the wavelength region of $20 \mathrm{~nm}$ at approximately $1.55 \mu \mathrm{m}$, and $20 \mathrm{~dB}$ was obtained in the region of $60 \mathrm{~nm}$. When the gap of the coupler was optimized at the respective wavelengths, the maximum extinction ratio was obtained, which was similarly to the result at $1.55 \mu \mathrm{m}$.

Although the approximate characteristics of the proposed optical switch were investigated using the analytical equations shown above, the properties of the switch were quantitatively and thoroughly simulated using electromagnetic software. We also performed a simulation using the finite difference time domain method (Crystal Wave) to confirm the mode profile and coupling. Figure 4 shows examples of the simulation results. Figure 4 a shows the intensity distribution of a
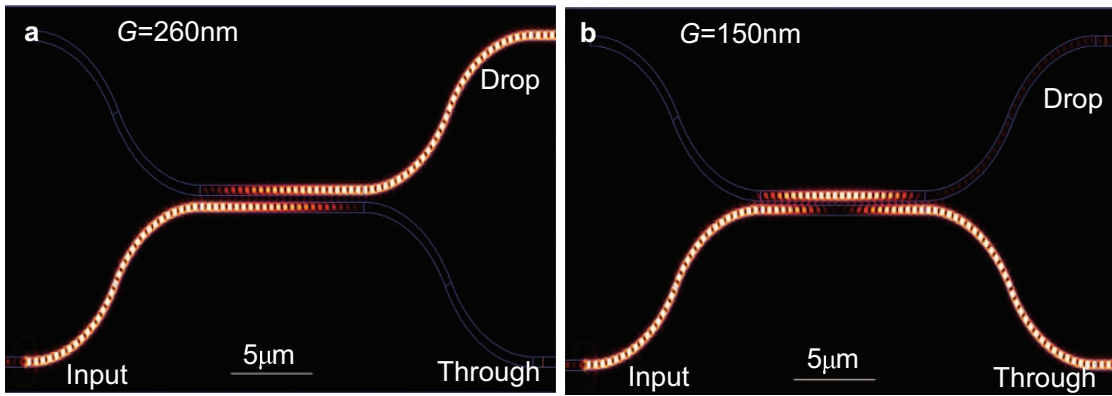

Figure 4 Simulated light wave propagation: (a) light wave intensity within the waveguides of the coupler switch at the first switch point with a gap $G$ value of $260 \mathrm{~nm}$; (b) the light wave intensity for a gap of $150 \mathrm{~nm}$. 


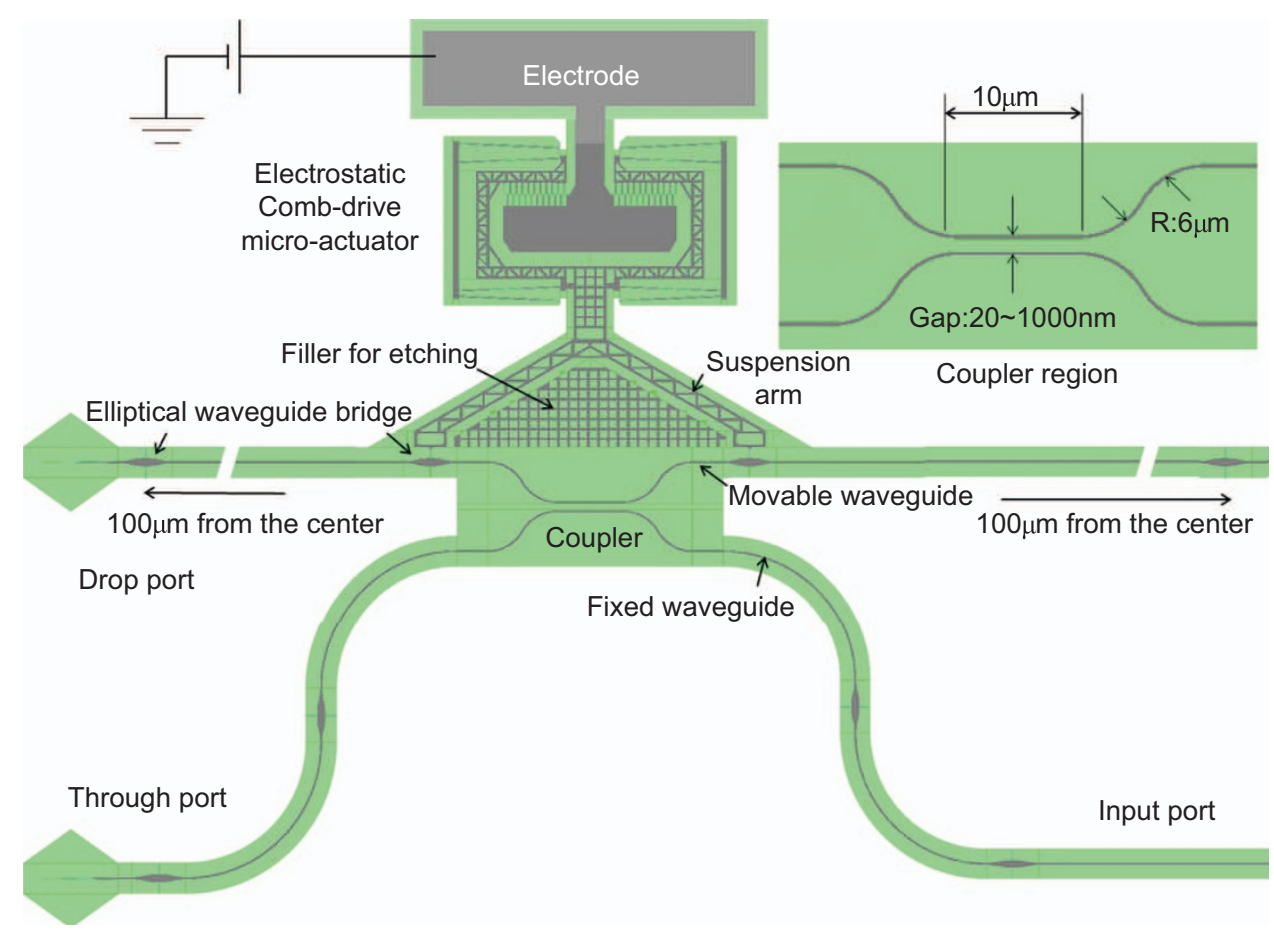

Figure 5 Mask pattern for the $2 \times 2$ waveguide coupler switch.

light wave propagating in the switch region at the first switch point $(G=260 \mathrm{~nm})$. The input light wave was clearly transmitted from the first waveguide to the second waveguide at a wavelength of $1.55 \mu \mathrm{m}$ with a negligible loss. The value of the gap at the first switch point was $65 \mathrm{~nm}$ larger than that obtained by the analytical equation. As the gap decreased from the condition presented in Figure $4 \mathrm{a}$, the light transferred from the first waveguide to the second waveguide again returned to the first waveguide at a gap of $150 \mathrm{~nm}$, as shown in Figure $4 \mathrm{~b}$. Based on these simulations, the coupling length corresponding to the straight region of the waveguide was designed to be $10 \mu \mathrm{m}$ in the gap region from $100 \mathrm{~nm}$ to $300 \mathrm{~nm}$ for switching. The switching properties of the proposed switch were explained well by the simulation, although the characteristic values shifted.

\section{FABRICATION AND EXPERIMENTATION}

Figure 5 shows the mask pattern of the proposed single switch. The length of the waveguides that were parallel was designed to be $10 \mu \mathrm{m}$. The gap was decreased from $1000 \mathrm{~nm}$ to $20 \mathrm{~nm}$ by the actuator. The movable freestanding silicon waveguide was suspended by $1.3-\mu \mathrm{m}-$ wide and $8.0-\mu \mathrm{m}$-long elliptical waveguide bridges, which connected the movable waveguide to the suspension arms of the actuator with $0.2-\mu \mathrm{m}$-wide and 1.6- $\mu \mathrm{m}$-long silicon beams. The distance between the two waveguide bridges was $52 \mu \mathrm{m}$. A mesh structure was patterned in the triangular region between the arms, which was a filler pattern used to prevent an excess dose of the electron beam. The filler pattern fell down onto the silicon substrate after etching the $\mathrm{SiO}_{2}$ layer. The movable waveguide was fixed to the substrate at a position $100 \mu \mathrm{m}$ from the coupler on both sides by the elliptical waveguide bridges, as shown in Figure 5. Therefore, the movable waveguide was supported by the two elliptical waveguide bridges at both ends, and the coupler was connected to the actuator. The other freestanding waveguide of the coupler was also fixed to the substrate by the elliptical waveguide bridges, as shown in Figure 5. These waveguides were $260 \mathrm{~nm}$ in thickness and $400 \mathrm{~nm}$ in width. The coupling part of the coupler was connected to two bent waveguides with a radius of $6 \mu \mathrm{m}$. The green region in Figure 5 was etched on the upper silicon layer of the SOI wafer. The width of the air-clad waveguide was $4.5 \mu \mathrm{m}$ and was held constant along the freestanding silicon waveguide. Therefore, the distances from the silicon waveguide to the surrounding upper silicon layer were $2.05 \mu \mathrm{m}$ on both sides of the waveguide. Certain minor modifications were added in a series of fabrications. It was desirable to keep the air-clad width constant along the silicon waveguide. For connecting the single switches in series, one waveguide bridge was needed to fix the movable waveguide between the switches.

The actuator consists of a comb drive with 20 finger pairs. Each finger was $1.73 \mu \mathrm{m}$ long and $200 \mathrm{~nm}$ wide. The actuator used doublefolded springs, and each beam of the springs was $200 \mathrm{~nm}$ in width and $15 \mu \mathrm{m}$ in length. The total spring constant of the actuator was calculated to be $0.315 \mathrm{~N} \mathrm{~m}^{-1}$. The electrostatic force of the actuator was also theoretically estimated to be approximately $0.32 \mu \mathrm{N}$ at $23 \mathrm{~V}$. The maximum displacement of the actuator was limited by a stopper at a displacement of $980 \mathrm{~nm}$. Therefore, the minimum gap of the coupler was designed to be $20 \mathrm{~nm}$. Voltage was applied between the two regions of the top silicon layer that were separated by etching. The movable waveguide was laterally deformed during switch operation. The spring constant of the movable waveguide for horizontal bending was less than that of the actuator by a factor of 75 . The vertical deformation of the suspended structure due to gravity was calculated to be less than $0.1 \mathrm{~nm}$. In the case of vibration with 10 times the acceleration of gravity, the displacement was as small as $1 \mathrm{~nm}$, which was also similar to the lateral vibration. Moreover, because the resonant frequency of the device was as high as $100 \mathrm{kHz}$, the proposed switch was not significantly affected by environmental vibrations.

A multiple switch was also designed and fabricated by connecting five $2 \times 2$ waveguide coupler switches in series and in parallel to investigate the feasibility of a matrix switch, as shown in Figure 1c. Each $2 \times 2$ 

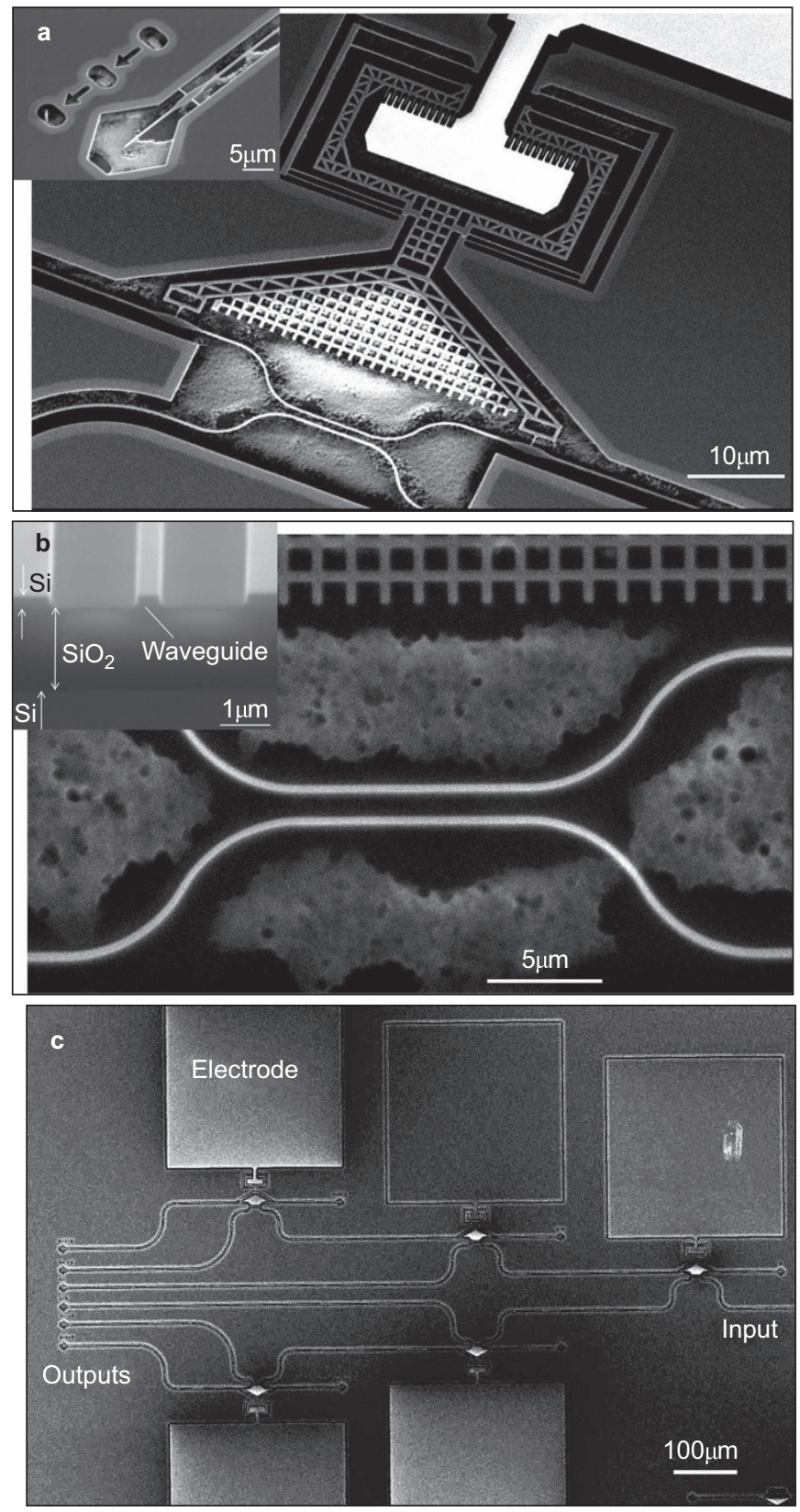

Figure 6 Electron micrographs of freestanding silicon waveguide coupler switches: (a) overview of the entire single switch with an inset showing the elongated output waveguide; (b) magnified view of the coupler region with an inset showing a cross-sectional image of a waveguide prior to $\mathrm{SiO}_{2}$ etching; (c) a $2 \times 6$ multiple switch

switch was connected with a $200-\mu \mathrm{m}$-long straight waveguide to form a single $2 \times 6$ multiple switch.

In the fabrication process, an SOI wafer with 260-nm-thick upper silicon layer and $2-\mu \mathrm{m}$-thick buried oxide layer on a $625-\mu \mathrm{m}$-thick silicon substrate was used. First, a 350 -nm-thick positive resist polymer (ZEON ZEP-520A) was coated onto the SOI wafer, which was then exposed using an electron beam patterning machine (JEOL JBX5000LS). After the development of the resist polymer, the top silicon layer was etched using a fast atom beam (Ebara FAB-60ML). The resist polymer was removed using an $\mathrm{H}_{2} \mathrm{SO}_{4} / \mathrm{H}_{2} \mathrm{O}_{2}$ solution. The SOI wafer was cleaved after partially dicing the silicon substrate to produce a facet on the input waveguide for light coupling. Finally, the $\mathrm{SiO}_{2}$ layer was etched using hydrofluoric acid vapor to obtain the freestanding structure of the proposed device.

The device characteristics were measured under an infrared optical microscope. A tunable laser (Agilent $81682 \mathrm{~A}$ ) was used as a light source at a wavelength of approximately $1.55 \mu \mathrm{m}$. A lensed singlemode fiber was used to couple the laser light to the end surface of the input port of the fabricated device. The light intensities at the output ports were measured based on spot images of scattered light at the ends of the waveguides using an infrared (IR) camera (Goodrich SU32KTS-1.7RT). ${ }^{10}$

\section{RESULTS AND DISCUSSION}

Figure 6a shows an electron micrograph of the fabricated waveguide switch. The freestanding silicon waveguides were fabricated, in close accordance with the designed specification. The movable waveguide was connected to an electrostatic comb-drive actuator, and the movable part of the actuator was freely suspended by silicon springs. The electrode for the fixed comb of the actuator appears white because the electrode was isolated and charged by the electron-beam scanning. The filler of the patterning was also charged after falling onto a residual $\mathrm{SiO}_{2}$ layer, as shown in Figure 6a. The inset of Figure 6a presents an oblique view of an output waveguide, which confirms that the waveguide was suspended in air. Figure $6 \mathrm{~b}$ shows a magnified image of the coupler region. Although a portion of the residual $\mathrm{SiO}_{2}$ layer is apparent in Figure $6 \mathrm{~b}$, the narrow freestanding silicon waveguides were uniformly formed by the proposed fabrication processes. The gap between the waveguides in the coupler region was approximately $100 \mathrm{~nm}$ larger than the design value, and the waveguide was approximately 5\% narrower than the designed value. The roughness of the sidewall of the waveguides, estimated based an electron micrograph, was less than $15 \mathrm{~nm}$. The inset of Figure $6 \mathrm{~b}$ shows a cross-sectional image of a waveguide prior to $\mathrm{SiO}_{2}$ etching, which confirms the rectangular shape of cross-section of the waveguide. Figure $6 c$ shows the fabricated $2 \times 6$ multiple switch comprising five $2 \times 2$ waveguide switches. The size of the multiple switch was not minimized because the electrode pads (250- $\mu \mathrm{m}$ square) for the actuators were enlarged for easy contact.

The switch characteristics were measured using a single $2 \times 2$ waveguide switch designed as shown in Figure 5. The output intensities were measured based on IR images obtained for different voltages applied to the actuator. The power of the laser source was $3 \mathrm{~mW}$, and the wavelength was $1.55 \mu \mathrm{m}$. The actuator voltage was varied from $0 \mathrm{~V}$ to $30.5 \mathrm{~V}$. Figure $7 \mathrm{a}-\mathrm{d}$ shows images of the output region. Figure $7 \mathrm{a}$ shows an image without the application of a voltage, which corresponds to a gap equal to $1000 \mathrm{~nm}$. Under this condition, the coupling between the waveguides was negligible, and a bright spot was observed at the through port. At $25.30 \mathrm{~V}$ (Figure $7 \mathrm{~b}$ ), the intensity at the through port was minimized, and the intensity at the drop port was maximized. This condition corresponds to the first switch point, at which the gap was approximately $250 \mathrm{~nm}$. As shown in Figure 7c, the second switch point occurred when the voltage was increased to $27.75 \mathrm{~V}$, at which the estimated gap was $130 \mathrm{~nm}$. A clear spot image was observed at the second switch point. With a further increase in the voltage, the output intensity at the drop port was again maximized, as shown in Figure 7d; the gap under this condition was approximately $70 \mathrm{~nm}$.

The spot images from the output ports were converted to intensity values, and the output intensity values at the through and drop ports were plotted as a function of the displacement, as shown in Figure 7e. The gap obtained based on the voltage dependence of the actuator 

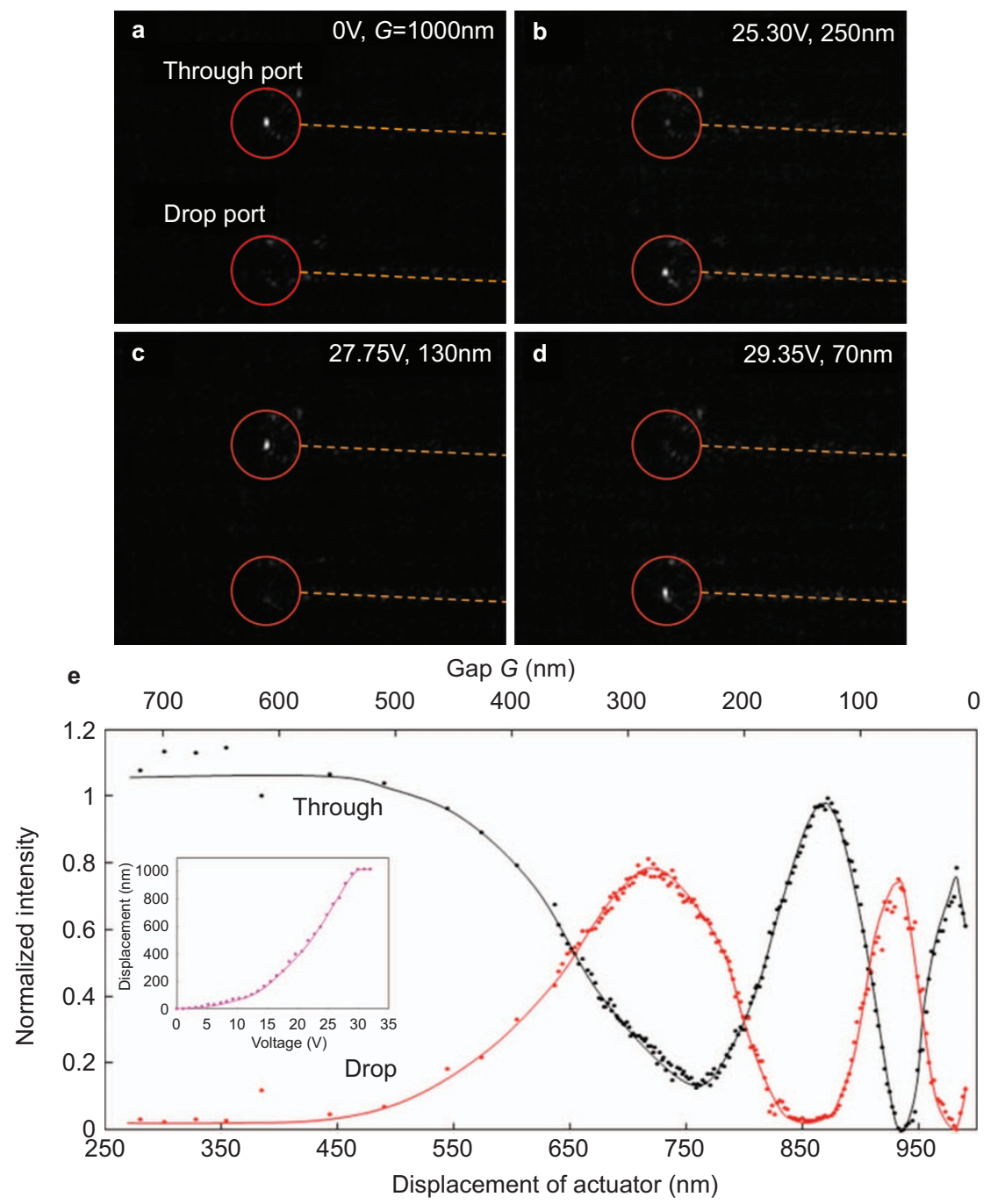

Figure 7 Infrared images of the output ports (a) at actuator voltages of $0 \mathrm{~V}$, (b) $25.30 \mathrm{~V}$, (c) $27.75 \mathrm{~V}$ and (d) $29.35 \mathrm{~V}$; (e) normalized output intensities at the through and drop ports measured as a function of the actuator displacement. The inset shows the voltage dependence of the actuator displacement.

displacement is also shown (inset in Figure 7e). The actuator displacement was measured by observing the actuator motion using a scanning electron microscope. The displacement rate was approximately $50 \mathrm{~nm} \mathrm{~V} \mathrm{~V}^{-1}$ at voltages greater than $20 \mathrm{~V}$. Because the minimum voltage setting was $\pm 0.01 \mathrm{~V}$, the resolution of the actuator was approximately $1 \mathrm{~nm}$, which corresponds to a signal fluctuation of less than $2 \%$. As shown in Figure 7e, oscillating intensities with opposite phases were obtained at the through and drop ports. At the first switch point of the $250-\mathrm{nm}$ gap, the maximum output intensity at the drop port was $20 \%$ less than that at the original intensity at the through port. However, the output intensity at the through port recovers to be slightly less than the original to the original intensity under the coupling condition of the double transition shown in Figure $4 \mathrm{~b}$ at a gap of $130 \mathrm{~nm}$, which generally agreed with the gap of $150 \mathrm{~nm}$ obtained in the simulation. Although the characteristic gaps calculated using the analytical equations were smaller than the experimental values, the relative dependence of the output intensities can be explained by the theoretical approach described in the section on 'Theoretical approach'.

The port extinction of the $2 \times 2$ switch was defined as $I_{1 \max } / I_{1 \min }$ and $I_{2 \max } / I_{2 \min }$, where $I_{1 \max }, I_{1 \min }, I_{2 \max }$ and $I_{2 \min }$ were the maximum and minimum intensities at port 1 (through port in this experiment) and port 2 (drop port), respectively. The port isolation of the $2 \times 2$ switch was the intensity ratio between the ports under the worst-case condition, which was defined as $\left(I_{2}\right.$ at $\left.I_{1 \mathrm{~min}}\right) / I_{1 \mathrm{~min}}$ at the first switch point in this experiment. The port extinction at the first switch point was $7.2 \mathrm{~dB}$, and the port isolation was $5.8 \mathrm{~dB}$. These low ratios were caused by the low intensity difference between the two ports at the first switch point and the position difference between the maximum point at the drop port and the minimum point at the through port. The reason for the differences is unclear. Because a large difference in the intensity between the ports was obtained at the second switch point, the above differences at the first switch point may have been caused by a position-dependent change in the waveguide impedance. Certain minor modifications in the waveguide arrangement generated a light scattering effect within the device. The ends of the waveguides were elongated to emit light and avoid reflection, as shown in Figure 5. The emission efficiency may have been affected by a small fabrication error due to the elongated structure, which may have caused different losses for the respective switch states, as seen in Figure 7e. A circular end of the waveguide may be better suited for uniform scattering. To optimize the switch characteristics as a function of the motion of 

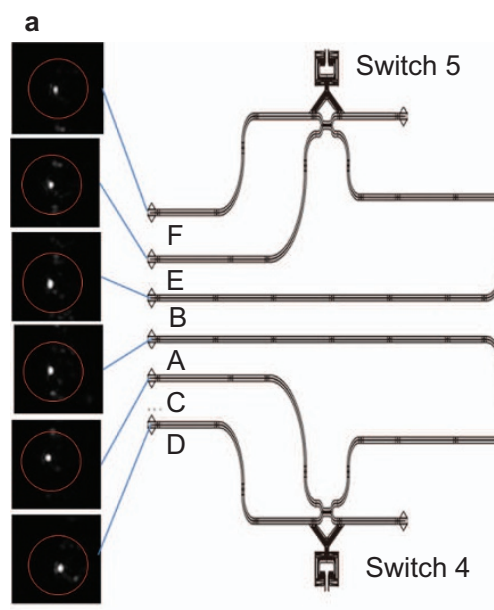

\section{Switch 3}
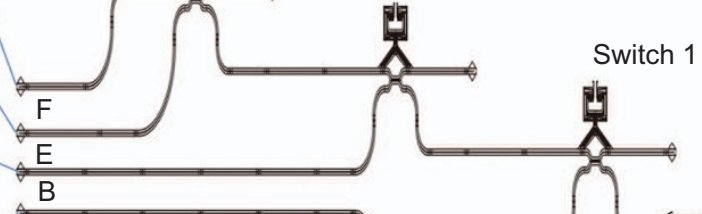

witch 1
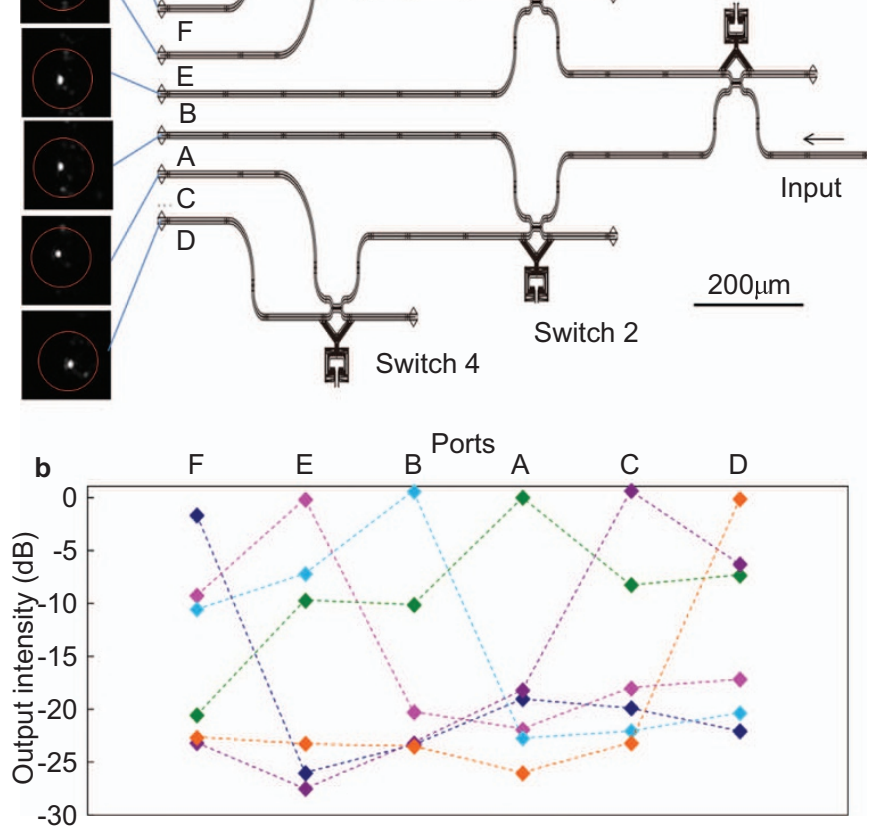

Figure 8 (a) Spot images at respective output ports of a multiple switch (left column) with a schematic layout of a $2 \times 5$ switch; (b) optical intensities at the respective ports when one of the outputs is in the on-state.

the actuator, further optical simulations of the entire structure of the switch, including the surrounding structures such as the clad and substrate, are needed.

The multiple switch consisting of five $2 \times 2$ switches shown in Figure $6 \mathrm{c}$ was also tested in the experiment. A minor modification of the $2 \times 2$ switch was introduced. The filler used for etching was changed to an island structure, and the etched region for the air-clad waveguide was minimized in the coupler region. The wavelength and power of the laser source used for the measurement were $1.55 \mu \mathrm{m}$ and $3 \mathrm{~mW}$, respectively. Figure 8 shows the results of a switch test using the fabricated multiple switch. In the test, input light was incident at the input of the first switch (Switch 1), and spot images at the respective output ports A-F were observed using an IR camera. Switches 1-5 were selectively turned on to construct an optical path to one of the output ports from A to F. Therefore, one of the six ports had an onstate (bright spot), and the others had an off-state (no spot). In the left column of Figure 8a, spot images are shown at the circle centers for the respective ports with a schematic diagram of the multiple switch. In the case of the output image at port A, a bright spot was observed after passing via two through ports of switches 1 and 2 . In the case of port $\mathrm{B}$, the light wave passes a drop port of switch 1 and a through port of switch 3. When port B was compared to port A, a similar bright spot was observed at port B, as shown in Figure 8a. A spot image similar to that at port $B$ was observed at port $C$, where the light wave passed three switches, including one drop port and two through ports. In the cases of ports $\mathrm{D}$ and $\mathrm{E}$, the light wave passed three switches, including two drop ports and one through port, and spots with slightly weaker intensities were observed. In the case of port $\mathrm{F}$, the intensity was weaker than that of others. Figure $8 \mathrm{~b}$ shows the measured output intensity values at the respective ports under the conditions shown in Figure 8a. The switch condition was selected to be equal to the condition of the port isolation measurement of a $2 \times 2$ switch at the first switch point, which corresponds to the worst case for all of the switching states of a $2 \times 6$ switch. The output differences among the respective ports during the on-states were within $2.3 \mathrm{~dB}$. The port isolation ranged from $6.2 \mathrm{~dB}$ to $27.3 \mathrm{~dB}$ for the adjacent ports and for the ports other than the adjacent ports, and the worst port isolation was $6.2 \mathrm{~dB}$ at port $\mathrm{D}$ between ports $\mathrm{C}$ and $\mathrm{D}$. The cross talk ranged from $6.9 \mathrm{~dB}$ to $28.1 \mathrm{~dB}$, and the worst value was obtained at port $\mathrm{D}$ when port $\mathrm{C}$ was at the on-state.

Based on the experiments described above, the insertion loss of the $2 \times 2$ switch was also roughly estimated to be approximately $1 \mathrm{~dB}$ on average. The switching time was estimated to be less than $50 \mu \mathrm{s}$ because the resonant frequency of the single switch was approximately $214 \mathrm{kHz}$. These experiments demonstrate that a matrix switch is feasible, although the optical arrangement is not optimized.

\section{CONCLUSIONS}

We investigated single and multiple silicon waveguide switches that employ gap-variable couplers with microelectromechanical combdrive actuators. The waveguide was $400 \mathrm{~nm}$ in width and $260 \mathrm{~nm}$ in thickness, and the size of the coupler switch was $50 \mu \mathrm{m}$ wide and $80 \mu \mathrm{m}$ long. The proposed switch was characterized using analysis based on the coupled-mode theory for rectangular waveguides as well as a simulation employing the finite difference time domain method. The two main switch points were found for gaps smaller than $260 \mathrm{~nm}$. A $2 \times 2$ single switch with an initial gap of $1000 \mathrm{~nm}$ and a $2 \times 6$ multiplex switch composed of five $2 \times 2$ switches were designed and fabricated using a combination of electron beam lithography, fast-atom beam etching and hydrofluoric acid vapor sacrificial etching. In an experiment, the properties of the single switch were measured in the gap region from $20 \mathrm{~nm}$ to $1000 \mathrm{~nm}$. Two switch points were clearly observed, and the relative gap dependence of the output intensities was explained by theoretical calculations. The port extinction was $7.2 \mathrm{~dB}$, and the port isolation was $5.8 \mathrm{~dB}$. The operation of the $2 \times 6$ switch was also confirmed in the experiment. The worst port isolation value was $6.2 \mathrm{~dB}$, and the worst cross talk value was $6.9 \mathrm{~dB}$; these values were generated at adjacent ports. The insertion loss of the $2 \times 2$ switch was estimated to be approximately $1 \mathrm{~dB}$ on average. To improve these characteristic values, it is necessary to further minimize fabrication errors on a nanometer scale and to stabilize the yield of the devices. It will also be useful to design actuators with a low displacement rate per voltage around the switch point to more precisely control the switch position. In addition, it will be useful to simulate the entire waveguide arrangement with actuator motion using rigorous electromagnetic calculations. Moreover, the background intensity is included in the output intensity measurements based on spot images obtained by an IR camera, and the dynamic range of the output values is small. Optical fiber detection is preferable for future measurements. The switching characteristics, such as the port isolation and extinction ratio, must be further improved (greater than $40 \mathrm{~dB}$ ) to be comparable to a commercialized silica matrix switch. However, because the insertion loss was relatively low $(\sim 1 \mathrm{~dB})$ in the multiple switch experiment, series connections of a few single switches are feasible for a matrix switch after improving the characteristics of a single switch. Due to its capacitive operation, the power consumption of the proposed waveguide switch was negligible compared to switches that use thermooptical effects. Therefore, the proposed switches are suitable for use in integrated silicon photonic circuits. 


\section{ACKNOWLEDGMENTS}

The authors thank Y. Kanamori and Y. Munemasa for their useful advice regarding the experiment and simulation. This work was supported by Strategic Information and Communication R\&D Promotion Program of Japanese Government. The device fabrication was performed at the Micro/ Nanomachining Research and Education Center of Tohoku University.

1 Jalali B, Fathpour S. Silicon photonics. J Lightwave Technol 2006; 24: 4600-4615.

2 Janz S, Cheben P, Dalage D, Densmore A, Lamontague B et al. Microphotonic elements for integration on the silicon-on-insulator waveguide platform. IEEE J Sel Top Quantum Electron 2006; 12: 1402-1415.

3 Yamada H, Chu T, Ishida S, Arakawa Y. Optical directional coupler based on Si-wire waveguides. IEEE Photon Technol Lett 2005; 17: 585-587.

4 Koonath P, Indukuri T, Jalali B. Monolithic 3-D silicon photonics. J Lightwave Technol 2006; 24: 1796-1804.

5 Yamada $\mathrm{H}$, Chu T, Ishida S, Arakawa Y. Si photonic wire waveguide devices. IEEE J Sel Top Quantum Electron 2006; 12: 1371-1379.

6 Green WM, Rooks MJ, Sekaric L, Vlasov YA. Ultra-compact, low RF power $10 \mathrm{~Gb} / \mathrm{s}$ silicon Mach-Zehnder modulator. Opt Express 2007; 15: 17106-17113.

7 Bulgan E, Kanamori Y, Hane K. Submicron silicon waveguide optical switch driven by microelectromechanical actuator. Appl Phys Lett 2008; 92: 101110.

8 Yao J, Leuenberger D, Lee MCM, Wu MC. Silicon microtoroidal resonators with integrated MEMS tunable coupler. IEEE J Sel Top Quantum Electron 2007; 13: 202-208.

9 Takahashi K, Kanamori Y, Kokubun Y, Hane K. A wavelength-selective add-drop switch using silicon microring resonator with a submicron-comb electrostatic actuator. Opt Exp 2008; 16: 14421-14428.

10 Ikeda T, Takahashi K, Kanamori Y, Hane K. Phase-shifter using submicron silicon waveguide coupler with ultra-small electro-mechanical actuator. Opt Exp 2010; 18: 7031-7037.
11 Chew X, Zhou G, Chau FS, Deng J. Nanomechanically tunable photonic crystal resonators utilizing triple-beam coupled nanocavities. IEEE Photon Technol Lett 2011; 23: 1310-1312.

12 Chew X, Zhou G, Chau FS, Deng J, Tang X et al. Dynamic tuning of an optical resonator through MEMS-driven coupled photonic crystal nanocavities. Opt Lett 2010; 5: 2517-2519.

13 Pruessner MW, Amarnath K, Datta M, Kelly DP, Kanakaraju S et al. InP-based optical waveguide MEMS switches with evanescent coupling mechanism. J Micromech Syst 2005; 14: 1070-1080.

14 Lee MC, Hah DD, Lau EK, Toshiyoshi H, Wu M. MEMS-actuated photonic crystal switches. IEEE Photon Technol Lett 2006; 18: 358-360.

15 Akihama Y, Kanamori Y, Hane K. Ultra-small silicon waveguide coupler switch using gap-variable mechanism. Opt Exp 2011; 19: 23658-23663.

16 Chang CC, Chiu WC, Wu JM, Lee MC, Shieh JM. MEMS-actuated waveguide phase modulator. In: Proceedings of the International Conference on Optical MEMS and Nanophotonics (OPT MEMS); 9-12 August 2010; Sapporo, Japan. IEEE: Piscataway, NJ, USA, 2010, pp99-100.

17 Fukazawa T, Hirano T, Ohno F, Baba T. Low loss intersection of Si photonic wire waveguides. Jpn J Appl Phys 2004; 43: 646-647.

18 Dutta AK, Dutta NK, Fujiwara M. WDM Technologies: Passive Optical Component II (6.6.Non-blocking Matrix Switch). Amsterdam: Academic Press; 2003. pp181-204.

19 Chai TY, Cheng TH, Bose SK, Lu C, Shen G. Array interconnection for rearrangeable 2-D MEMS optical switch. J Lightwave Technol 2003; 21: 1134-1140.

20 Marcatili EAJ. Dielectric rectangular waveguide and dielectric coupler for integrated optics. Bell Syst Tech J 1969; 47: 2071-2102.

21 Okamoto K. Basis of Optical Waveguides. Tokyo: Coronasha Ltd; 1992.

This work is licensed under a Creative Commons Attribution-NonCommercial-NoDerivative Works 3.0 Unported License. To view a copy of this license, visit http:// creativecommons.org/licenses/by-nc-nd/3.0 